EPJ Web of Conferences 38, 01002 (2012)

DOI: $10.1051 /$ epjconf/20123801002

(C) Owned by the authors, published by EDP Sciences, 2012

\title{
Research at SHIP - status and perspectives
}

\author{
Sophie Heinz ${ }^{1, \text { a }}$ for the SHIP collaboration \\ GSI Helmholtzzentrum für Schwerionenforschung, Planckstrasse 1, 64291 Darmstadt, Germany
}

\begin{abstract}
The superheavy element program at the velocity filter SHIP at GSI focused during recent years on hot fusion reactions with ${ }^{248} \mathrm{Cm}$ targets. In collisions of ${ }^{48} \mathrm{Ca}+{ }^{248} \mathrm{Cm}$ a total of six decay chains was observed which were attributed to the isotopes ${ }^{292} 116$ and ${ }^{293} 116$. Further, the reaction ${ }^{54} \mathrm{Cr}+{ }^{248} \mathrm{Cm}$ was applied to search for isotopes of the element $Z=120$. Besides the experimental program, we plan a mid-term upgrade of SHIP and develop new detection techniques.
\end{abstract}

\section{Introduction}

Until the year 2005, so-called cold fusion reactions using doubly magic $\mathrm{Pb}$ and $\mathrm{Bi}$ targets were applied at SHIP for the synthesis of superheavy elements and lead to the discovery of the elements $Z=107-112$ [1]. Starting in 2005 , hot fusion reactions with actinide targets were introduced at SHIP. This was motivated by experimental results from the Dubna gas-filled separator (DGFRS) at the Flerov Laboratory of Nuclear Reactions where relatively large cross-sections on the order of $1 \mathrm{pb}$ were observed for nuclei with $\mathrm{Z}=114-118$ in hot fusion [2]. In contrast, the cross-sections in cold fusion reactions drop by about one order of magnitude for every two protons more in the compound nucleus $(\mathrm{CN})$ and become already as low as $30 \mathrm{fb}$ for $Z=113$ [3]. The application of hot fusion reactions at SHIP is an important step not only for the synthesis of new elements with $Z>118$ but equally for the confirmation of existing data from hot fusion on $112 \leq \mathrm{Z} \leq 118$. The latter have been synthesized until recently only at gas-filled separators, mainly in Dubna. SHIP is presently the only velocity filter applied for the synthesis of the heaviest elements with exception of the energy filter VASSILISSA [4] in Dubna. Therefore, the cross-sections and decay properties of superheavy nuclei measured at SHIP represent an important cross-check of the existing data with respect to possible systematic errors.

Additionally, SHIP allows the identification of the reaction channel (xn, $\alpha \mathrm{xn}$, transfer etc.) with good resolution by measuring the velocity spectra of the reaction products [5]. The knowledge of the reaction channel is crucial for assigning an observed decay chain to the correct mother isotope since the mass and nuclear charge of the superheavy nuclei are not measured directly. Moreover, the good separation between different reaction channels enables also the study of other reactions than fusion. We use this feature, for example, for the study of multi-nucleon transfer reactions. One point of interest is whether this reaction type can be applied to synthesize new neutron-rich heavy and superheavy isotopes. There, the region of $\mathrm{N}=126$ and the region of $Z>92$ are of special interest. Neutron-rich isotopes around $\mathrm{N}=126$ are usually produced in fragmentation reactions but new theoretical calculations result in

\footnotetext{
a e-mail: s.heinz@gsi.de
}

larger cross-sections for deep inelastic transfer reactions (see e.g. [6,7]) while the neutron-rich region at $Z>92$ is not reachable in other reactions.

The production cross-sections of superheavy nuclei are small and reach the sub-picobarn region for the heaviest known isotopes. This presently limits the synthesis of new elements with the available beam intensities of several $10^{12}$ ions per second. Reaction cross-sections of $100 \mathrm{fb}$ require several months of beam time to reach the one-event crosssection limit for the desired isotope. Therefore, the availability of higher beam intensities and the related necessary upgrades of the separation and detection techniques are decisive steps for the future research in this field. As a midterm project we plan a technical upgrade of SHIP where the main point is an increase of the angular acceptance. As a long-term project, GSI plans a new superconducting continuous wave LINAC [8] for ion beams at Coulomb barrier energies. The beam intensities are expected to be 10 to 50 times larger than the presently available currents. Within this frame, we are developing a concept for a new separator which allows a strong suppression of primary beams with intensities up to $10^{14}$ particles per second. In parallel to the separator design, we are testing and developing new detection techniques for nuclei which are long-lived and/or undergo beta-decay or spontaneous fission and are therefore not accessible with the available techniques.

\section{Experimental setup}

A scheme of the velocity filter SHIP [9] and the detection system is shown in figure 1. SHIP is a Wien filter which separates the ions according to their velocities. Reaction products which leave the target at forward angles of $(0 \pm$ 2) degree with respect to the beam direction are accepted by the entrance aperture of SHIP. The electric and magnetic fields are chosen such that the relatively light and fast projectiles and projectile-like reaction products are deflected to the beam stop while the much slower evaporation residues pass SHIP and reach the focal plane detector. The accepted velocity window at a given setting is $\Delta \mathrm{v} / \mathrm{v}=0.1$ (FWHM). All reaction products which pass the velocity filter are implanted in a position sensitive 16 strip silicon detector ("stop detector") where their time of implantation, 


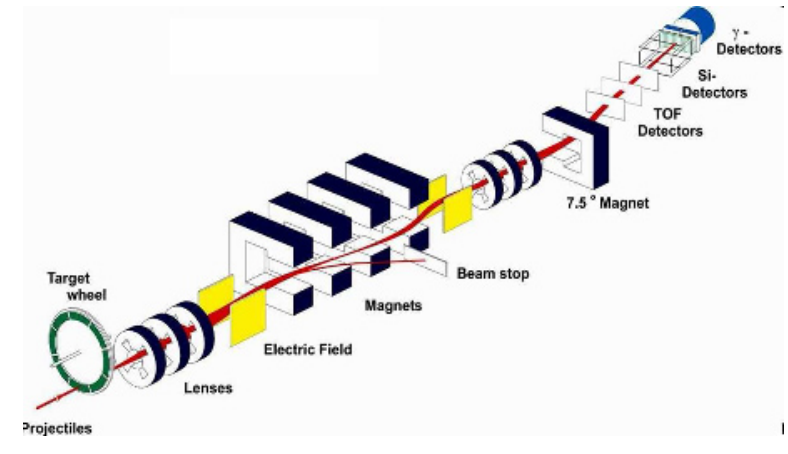

Fig. 1. The velocity filter SHIP and detection system. For details see text.

position, kinetic energy and radioactive decays are registered [10]. Especially the $\alpha$-decay properties allow for an unambiguous identification of single isotopes. Six further Si detectors are installed in a box-like arrangement ("box detector") in front of the stop detector and cover $85 \%$ of the backward hemisphere in order to register $\alpha$-particles and fission fragments escaping from the stop detector. Finally, a germanium clover detector is mounted behind the stop detector. It consists of four germanium crystals which register gamma rays from excited reaction products implanted in the stop detector. Three time-of-flight (TOF) detectors [11] are installed in front of the Si detectors. They fulfill two tasks: On one hand the measured TOF together with the energy depositon in the Si detector allows to distinguish between projectile-like nuclei, target-like nuclei and fusion evaporation residues. Secondly, they allow to distinguish between ions which have been produced in the target and radioactive decay products ( $\alpha$-particles or fission fragments) which have been created in the Si detector by decay of implanted mother isotopes. In the first case the particles have to pass the TOF detectors while in the latter case they don't. Further details concerning the setup can be found in [1]. The combination of single isotope identification via radioactive decays and the strong background suppression by the separator allows to reach cross-section limits of $10 \mathrm{pb}$ in one day of beam time with typical beam intensities of several $10^{12}$ particles per second. For additional suppression of $\alpha$-like and fission-like background events the beam-off periods are used which are provided by the pulsed structure of the beam consisting of $5 \mathrm{~ms}$ long beam-on periods followed by $15 \mathrm{~ms}$ long beam-off periods.

For the identification of the reaction channel (fusionevaporation, transfer, etc.) we measure the velocity spectra of the produced isotopes. To obtain the spectra, we vary the electric and magnetic field strength, E and B, of the velocity filter accordingly. The ration E/B determines at which velocity a particle can pass SHIP. The separation according to velocities leads to a better relative separation between different reaction channels in comparison to the separation according to magnetic rigidities $\mathrm{B} \rho$. For example, in the reaction ${ }^{48} \mathrm{Ca}+{ }^{248} \mathrm{Cm} \rightarrow{ }^{296} 116^{*}$ at $5.5 \mathrm{MeV} / \mathrm{u}$ the velocities of the $\mathrm{CN}$ and of the elastically scattered target ions differ by $100 \%$ while the respective $\mathrm{B} \rho$ values for the ions in gas differ by only $20 \%$.

\section{Hot fusion reactions with ${ }^{248} \mathrm{Cm}$ targets}

\subsection{The reaction ${ }^{48} \mathrm{Ca}+{ }^{248} \mathbf{C m} \rightarrow{ }^{296} 116^{*}$}

The nuclei with the largest proton numbers synthesized so far at SHIP, and also at GSI, are isotopes of element $\mathrm{Z}=116$ in reactions of ${ }^{48} \mathrm{Ca}+{ }^{248} \mathrm{Cm} \rightarrow{ }^{296} 116^{*}$ (June $25-$ July 26,2010$)$ [12]. The same reaction was already studied earlier at the Dubna gas-filled separator in several experiments during the years 2000 to 2004 at compound nucleus excitation energies of 33 and $39 \mathrm{MeV}[13,14]$. In the Dubna experiments five decay chains were observed which were attributed to the decay of the isotope ${ }^{293} 116$ ( $3 n$ evaporation channel) and six decay chains which were attributed to ${ }^{292} 116$ (4n channel). At SHIP, we continued the excitation function to higher energies of 41 and 45 $\mathrm{MeV}$. At $41 \mathrm{MeV}$ we observed six $\alpha$-decay chains of different lengths, all of them terminated by a spontaneously fissioning nucleus (figure 2). Four of the chains consist of an implanted recoil nucleus followed by two $\alpha$-decays and a fission event. The length of these chains as well as the half-lives and $\alpha$-energies of the chain members are well in agreement with the data measured in Dubna for the isotope ${ }^{292} 116$. Also the corresponding cross-section of $3.4 \mathrm{pb}$ well continues the excitation function measured in Dubna. Further, the measured cross-section of $3.4 \mathrm{pb}$ is well in agreement with the cross-section predicted in model calculations (3 pb) [15] for the same excitation energy. This good agreement between experimental data and theoretical expectations further supports the attribution of the four decay chains to the mother isotope ${ }^{292} 116$.

Besides, two further chains were observed. One of them consists of an implanted recoil nucleus followed by three $\alpha$-decays and a fission decay. This decay sequence, the $\alpha$ energies and half-lives are consistent with the data measured in Dubna for the isotope ${ }^{293} 116$ (3n channel). Also the related cross-section of $0.9 \mathrm{pb}$ agrees well with these data and with theoretical predictions. In the other decay chain the recoil nucleus was followed by four $\alpha$-decays and a fission event. In this chain only the energy of the first $\alpha$-particle was in agreement with the values measured in Dubna for the nucleus ${ }^{293} 116$. Therefore, we attributed this chain preliminary also to the mother isotope ${ }^{293} 116$. The last $\alpha$-decay in the chain with the energy of 9.315 $\mathrm{MeV}$ would then originate from an $\alpha$-branch of the isotope ${ }^{281}$ Ds. An $\alpha$-decay of ${ }^{281}$ Ds was so far only observed in an experiment at the gas-filled separator TASCA at GSI [16] however with an energy of $8.727 \mathrm{MeV}$ which is 588 $\mathrm{keV}$ less than the energy observed at SHIP. The half-lives of the four $\alpha$-decays observed at SHIP and attributed to ${ }^{293} 116 .,{ }^{289} 114,{ }^{285} \mathrm{Cn}$ and ${ }^{281} \mathrm{Ds}$ are in agreement with the literature values within statistical fluctuations. But the $\alpha$ decay energies of the daughter nuclei ${ }^{289} 114$ and ${ }^{285} \mathrm{Cn}$ are $211 \mathrm{keV}$ and $523 \mathrm{keV}$ larger than the literature values. A possible explanation for the deviating $\alpha$-energies observed at SHIP might be the population of isomeric states which are located rather close to the ground state. Theoretical calculations for the decay chain of ${ }^{293} 116$ [17] result in the existence of high and low spin states close to the ground state which would enable the existence of isomeric states. The observation of very similar half-lives for the decay chains from the hypothetical ground and isomeric states is not contradicting according to the calculations in [17] since they allow for the existence of decay chains from the 


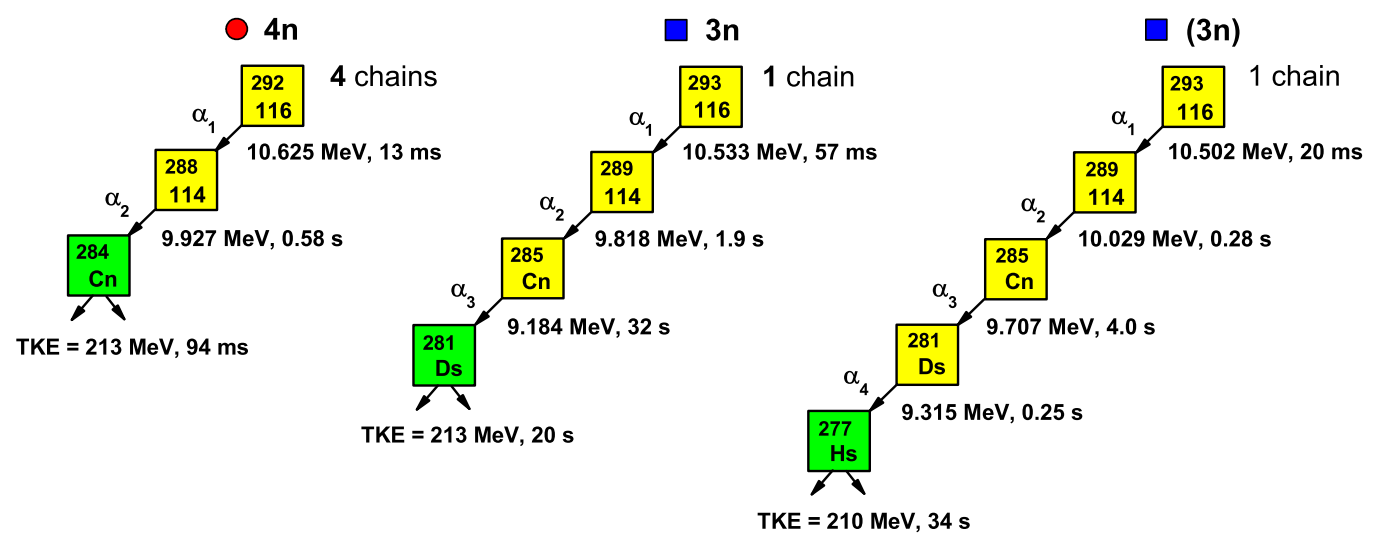

Fig. 2. Decay chains observed at SHIP in fusion reactions of ${ }^{48} \mathrm{Ca}+{ }^{248} \mathrm{Cm}$ leading to the compound nucleus ${ }^{296} 116^{*}$ at an excitation energy of $41 \mathrm{MeV}$. The given half-lives and $\alpha$-decay energies include all available data from different experiments. The decay sequence ( $\alpha-\alpha-\alpha-\alpha$-fission) was so far only observed at SHIP and preliminary attributed to the $3 \mathrm{n}$ evaporation channel. For this case the half-lives and decay energies represent the values measured at SHIP.
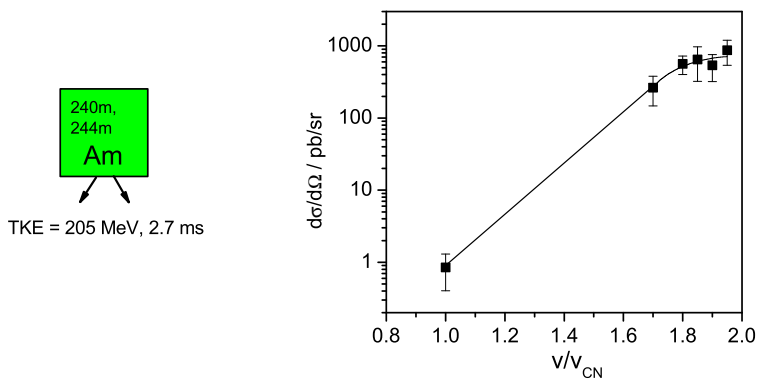

Fig. 3. left panel: Spontaneous fission decay observed in collisions of ${ }^{48} \mathrm{Ca}+{ }^{248} \mathrm{Cm}$. The SHIP settings were optimized for the transmission of fusion-evaporation residues of the compound nucleus ${ }^{296} 116^{*}$. right panel: velocity distribution of fission events with similar characteristics. The strong increase of the crosssection towards $\mathrm{v} / \mathrm{v}_{C N}=2.0$ points to a transfer process as their origin.

ground as well as from isomeric states with similar angular momentum leading to similar half-lives. However, an assignment of the observed transitions by comparing experimental and theoretical data is not possible.

In addition to the $\alpha$-decay chains we observed an implanted recoil nucleus at the excitation energy of $41 \mathrm{MeV}$ which was directly followed by a spontaneous fission decay after $2.7 \mathrm{~ms}$ (see figure 3). The total kinetic energy of the fission fragments was $205 \mathrm{MeV}$. A cross-section of $\left(0.9_{-0.7}^{+2.1}\right) \mathrm{pb}$ can be ascribed to this event. From terms of half-life, fission decay energy and cross-section the event could be interpreted easily as a fission branch of an isotope of element 116. However, fission decays of these nuclei should be strongly hindered due to the large expected fission half-lives on the order of $10^{6} \mathrm{~s}$ which would lead to fission branches of about $10^{-7}$. The origin of the observed decay was finally revealed by the velocity spectrum of the reaction products from ${ }^{48} \mathrm{Ca}+{ }^{248} \mathrm{Cm}$ collisions. At
SHIP settings for the transmission of nuclei with velocities around two times the $\mathrm{CN}$ velocity, $\mathrm{v}=2 \mathrm{v}_{C N}$, we observed a strong increase of the yield of fission events with very similar signatures (figure 3 ). The maximum of the velocity distribution was located close to $\mathrm{v}=2 \mathrm{v}_{C N}$. The respective production cross-section was three orders of magnitude larger than at the setting for nuclei with the $\mathrm{CN}$ velocity. The maximum close to $\mathrm{v} / \mathrm{v}_{C N}=2.0$ is a clear hint that the respective nuclei have been produced in transfer reactions after central collisions. Assuming a two-body process a total kinetic energy of $184 \mathrm{keV}$ (in the center-ofmass) of the transfer products in the exit channel can be deduced from the position of the velocity peak. This corresponds to $86 \%$ of the available center-of-mass energy of $214 \mathrm{MeV}$ which reveals that the nuclei have been produced in quasi-elastic transfer reactions. The quasi-elastic nature of the process indicates that the reaction products are located in the vicinity of the target nucleus ${ }_{96}^{248} \mathrm{Cm}$. The closest-lying and most probable candidates with appropriate half-lives are the fission isomers ${ }_{95}^{240 m} \mathrm{Am}\left(\mathrm{T}_{1 / 2}=0.94\right.$ $\mathrm{ms})$ and ${ }_{95}^{244 m} \mathrm{Am}\left(\mathrm{T}_{1 / 2}=0.9 \mathrm{~ms}\right)$ which can easily be populated by the transfer of one proton and a few neutrons. Also at settings which are optimized for the transmission of fusion-evaporation residues about one per mil of the target-like transfer products can pass the velocity filter and reach the focal plane detector. The reason is the low atomic charge states which can be adopted by a small fraction of the nuclei. Due to the resulting large magnetic rigidities they traverse the separator without noticeable deflection.

\subsection{Search for $Z=120$}

The reaction ${ }^{54} \mathrm{Cr}+{ }^{248} \mathrm{Cm}$ was studied at SHIP to search for evaporation residues of the compound nucleus ${ }^{302} 120^{*}$. This proton number is particularly interesting because in some theoretical models a spherical shell closure is expected there. The compound nucleus ${ }^{302} 120$ has 182 neutrons. This would be the closest approach to the predicted closed neutron shell $\mathrm{N}=184$ reached so far in superheavy 


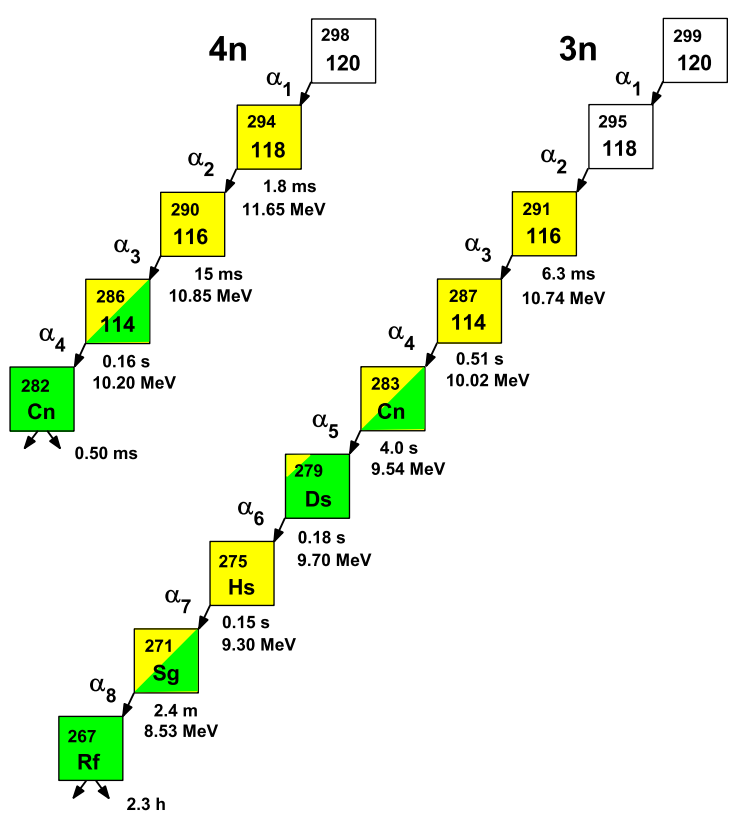

Fig. 4. Expected decay chains of the isotopes ${ }^{298} 120$ and ${ }^{299} 120$. The squares with white background represent still unobserved isotopes.

element experiments. Calculations within different models have been performed for this reaction which resulted in strongly different production cross-sections with values between $0.07 \mathrm{fb}$ [18] and $800 \mathrm{fb}$ [19] for the $4 \mathrm{n}$ evaporation channel. The strong variations between the results reflect the high sensitivity of the cross-sections to the entrance and exit channel parameters like fusion barriers and shell correction energies which vary in the different models or have different impact, respectively. One has to note that all models were able to reasonably reproduce the measured cross-sections for lighter superheavy nuclei. The models underlying the above given values assumed fission barriers of about $7 \mathrm{MeV}$ and a proton shell closure at $\mathrm{Z}=114$. However, if the fission barriers are actually higher and/or the shell closure is located at $\mathrm{Z}=120$, much higher crosssections can be expected. Concerning the strength of the shell corrections, the model predictions vary on a large scale resulting in values for the shell correction energies from $-6 \mathrm{MeV}$ to $-12 \mathrm{MeV}$ which corresponds to fission barriers of $(6-12) \mathrm{MeV}$ [20]. By trend, one can assume that the cross-section increases by one order of magnitude if the fission barrier increases by $1 \mathrm{MeV}$.

The first part of the experiment ${ }^{54} \mathrm{Cr}+{ }^{248} \mathrm{Cm}$ was performed in the time of April 23 to June $1^{s t}, 2011$ [21]. The beam energy was chosen such that the compound nuclei would be created with an excitation energy of $40 \mathrm{MeV}$ which should predominantly lead to the evaporation residues ${ }^{298} 120$ and ${ }^{299} 120$. From both isotopes long $\alpha$-decay chains can be expected which join the already known decay chains of ${ }^{294} 118$ and ${ }^{291} 116$, respectively (figure 4). Finally, no event was observed which showed the expected signatures of the decay of a $Z=120$ isotope. The upper cross-section limit reached in this experiment is $560 \mathrm{fb}$ at a total number of $7.9 \times 10^{18}$ projectiles on target. The experimental crosssection limit allows the exclusion of extraordinary high fis- sion barriers as predicted by some models. From the value of $560 \mathrm{fb}$ one can deduce that the fission barriers do not significantly exceed the value of $8 \mathrm{MeV}$.

The expected half-lives for isotopes with $\mathrm{Z}=120 \mathrm{de}$ serve special attention. The $\mathrm{Q}$-values for $\alpha$-decay are increasing with the proton number which leads to a decrease in the half-lives. According to the macroscopic-microscopic model which assumes the shell closure at $Z=114$ the Qvalues are about $13 \mathrm{MeV}$ leading to half-lives on the order of $(1-10) \mu$ s for the isotopes ${ }^{298,299} 120$ This is already on the same range as the flight time of the fusion products through the separator. Therefore one has to keep in mind that the evaporation residues could already decay before the detector is reached. If, however, the shell closure is assumed at $Z=120$ one can expect significantly longer halflives on the order of $1 \mathrm{~s}$. The related Q-values are around $11 \mathrm{MeV}$. Therefore, the observation of relatively long halflives and $\alpha$-decay energies around $11 \mathrm{MeV}$ would be a strong hint for a shell closure at $Z=120$.

\section{Technical developments}

As a mid-term project we plan an upgrade of SHIP which comprises an increase of the angular acceptance, a new deadtime free data acquisition system and additional instrumentation for the detection and identification of the reaction products. The larger angular acceptance will allow for a two to three times larger efficiency. This is especially important for very asymmetric fusion reactions with ratios between projectile and target mass of $\mathrm{m}_{p} / \mathrm{m}_{t}<0.2$. Further, it is equally important for experiments on transfer reactions. Deep inelastic heavy transfer products have usually very broad angular distributions with emission angles up to about 50 degree in the laboratory system which leads to strong losses at the entrance of SHIP. Presently, only a few percent of the target-like transfer products are accepted while after an acceptance increase an efficiency of about $10 \%$ can be reached. To reach the larger acceptance, we plan to replace the entrance and exit magnetic quadrupole triplets by stronger, superconducting triplets with a larger bore diameter. Additionally, the targets can be installed at a smaller distance from the entrance quadrupole triplet.

Further, the present data acquisition system with a conversion plus dead time of $20 \mu$ s will be replaced by a new digital system. The new system will consist of Pixie-16 modules [22] and will operate with effectively zero deadtime. The digital sampling is performed at $100 \mathrm{MHz}$. This allows to resolve the decays of very short-lived implanted nuclei with lifetimes on the scale of $(10-100)$ ns which is three orders of magnitude shorter than the presently accessible half-lives. A part of the Pixie electronics was already successfully running in the ${ }^{54} \mathrm{Cr}+{ }^{248} \mathrm{Cm}$ experiment. During this experiment, six Pixie-16 modules were employed in parallel to the present analog setup for the readout of the stop and box detector signals.

As a third point we plan an extension of the detection system. Presently, the identification of single nuclei is done via their $\alpha$-decays after implantation in the silicon detectors. This means that clear identification can only be done for $\alpha$-emitting nuclei with relatively short half-lives on the order of seconds or less. Concerning $\beta$-emitters, the system allows a restricted access, meaning, that the $\beta$-decay has to populate with sufficient branching an excited state 
in the daughter nucleus which then de-excites via gamma and X-ray emission. In this case, the mother and daughter nuclei are identified by the gamma energies. However, with respect to unambiguousness of identification and detection efficiency the method is less effective than the identification via $\alpha$-decays. As an additional means for particle identification we plan to perform isobaric identification by mass measurements. Penning traps or multiple reflection time-of-flight mass spectrometers have mass resolving powers up to $\mathrm{m} / \Delta \mathrm{m} \approx 10^{7}$ where, however, a resolving power of $10^{5}$ is already sufficient for an isobaric separation of most of the isotopes. The mass measurement is performed after the separation stage. Penning traps and TOF spectrometers can only trap ions with low energies on the scale of $(100-1000) \mathrm{eV}$. Therefore a buffer gas cell and an ion guide system has to be used after the separator for stopping, extracting and transporting the ions to the mass measurement device. The presently running gas cells which are applied for ions in the required mass and energy range have overall (i. e. stopping and extraction) efficiencies of about $1 \%$. This leads to a rather strong loss of ions which presently requires production cross-sections of at least $10 \mathrm{nb}$. However, the new generation of cryogenic gas cells [23] shows at least a factor of 10 more efficiency which has been demonstrated in the commissioning of such a cell at the Fragment Separator at GSI. Further, a newly developed multiple reflection time-of-flight mass spectrometer (MR-TOF-MS [24]) at the University of Giessen has promising features for the application as detection system since it allows for a broadband detection. This is especially for transfer products very efficient since a large number of them with rather different mass numbers can pass the separator at the same setting.

As a long-term project, GSI plans a new superconducting continuous wave (cw) LINAC which shall provide ion beams with intensities up to $10^{14}$ particles / s. Presently, a so-called demonstrator, consisting of a superconducting $\mathrm{CH}$-cavity and two superconducting solenoids is built and shall be tested with beam in 2013 / 2014 at GSI [8]. In parallel, we are developing a new separator for the future superheavy element program which will be adjusted to the requirements arising with the higher beam intensities, namely, it must be capable of strong background suppression to handle the 10 to 50 times higher beam intensities. The new separator will also be based on the concept of a velocity filter like the present SHIP, following from the positive long-term experience with SHIP. Velocity filters provide several advantages in comparison to gas-filled separators: (i) the separation according to velocities is, for the typical reactions applied here, about five times stronger than the separation according to magnetic rigidity in gas. Therefore, velocity filters provide a stronger suppression especially of target-like quasi-elastic and deep inelastic background events; (ii) velocity filters allow the determination of the reaction channel in which a certain isotope was created by measuring the velocity spectra of the reaction products; (iii) due to the relatively strong separation of different reaction channels, velocity filters are also suitable for the study of transfer reactions with low cross-sections, especially at beam energies below and close to the Coulomb barrier. The study of transfer reactions is very interesting since in recent times new theoretical calculations suggest to produce new neutron-rich heavy and superheavy nuclei in multi-nucleon transfer reactions which are not accessible in other reactions (see e.g. [25,26]) For the new separator we plan a more compact design in comparison to SHIP. It will also be a two-stage separator but the condenser field shall be placed inside the magnetic dipole field while SHIP has separated electric and magnetic fields.

\section{References}

1. S. Hofmann and G. Münzenberg, Rev. Mod. Phys. 72 (2000) 733.

2. Yu. Oganessian, J. Phys. G: Nucl. Part. Phys. 34 (2004) R165.

3. K. Morita et al., J. Phys. Soc. Jpn. 73 (2004) 2593.

4. A. Yeremin et al., Nucl. Instr. Meth. B 126 (1997) 329.

5. S. Heinz et al., Eur. Phys. Jour. A 48 (2012) 32.

6. G.G. Adamian et al., Phys. Rev. C 81 (2010) 057602.

7. V. Zagrebaev et al., J. Phys. G: Nucl. Part. Phys. 35 (2008) 125103.

8. S. Mickat et al., Proc. of the SRF Conference 2011, Chicago 2011.

9. G. Münzenberg et al., Nucl. Instr. Meth. 161 (1979) 65.

10. S. Hofmann et al., Z. Phys. A 291 (1979) 53.

11. S. Saro et al., Nucl. Instr. Meth. 381 (1996) 520.

12. S. Hofmann et al., Eur. Phys. Jour. A 48 (2012) 62.

13. Yu. Ts. Oganessian et al., Phys. Rev. C 63 (2000) 011311.

14. Yu. Ts. Oganessian et al., Phys. Rev. C 70 (2004) 064609.

15. V.I. Zagrebaev, Nucl. Phys. A 734 (2004) 164.

16. Ch.E. Düllmann et al., Phys. Rev. Lett 104 (2010) 252701.

17. S. Cwiok et al., Phys. Rev. Lett. 83 (1999) 1108.

18. G.G. Adamian et al., Eur. Phys. Jour. A 41 (2009) 235.

19. A.K. Nasirov et al., Phys. Rev. C 79 (2009) 024606.

20. M. Bender et al., Phys. Lett. B 515 (2001) 42.

21. S. Hofmann et al., GSI Sci. Rep. 2011, (2012) 205.

22. http://www.xia.com/DGF_Pixie-16.html

23. P. Dendoven et al., Nucl. Instr. Meth. A 558 (2006) 4488.

24. W.R. Plass et al., Nucl. Instr. Meth. B 266 (2008) 4560.

25. G.G. Adamian et al., Phys. Rev. C 71 (2005) 034603.

26. V. Zagrebaev and W. Greiner, Phys. Rev. C 78 (2008) 34610 